American Journal of Pharmacology and Toxicology 5 (1): 9-13, 2010

ISSN 1557-4962

(C) 2010 Science Publications

\title{
Risk Assessment of Tobacco Types and Oral Cancer
}

\author{
${ }^{1}$ Abdoul Hossain Madani, ${ }^{2}$ Abdolreza Sotoodeh Jahromi, \\ ${ }^{3}$ Madhurima Dikshit and ${ }^{4}$ Debanshu Bhaduri \\ ${ }^{1}$ Department of Hygiene, Hormozgan University of Medical Sciences, Bandarabbas, Hormozgan, Iran \\ ${ }^{2}$ Department of Immunology, Jahrom University of Medical Sciences, Jahrom, Iran \\ ${ }^{3}$ Department of Surgical Oncology, Morbai Naraindas Budhrani Cancer Institute, \\ Inlaks and Budhrani Hospital, Pune, India \\ ${ }^{4}$ Biochemistry Division, Department of Chemistry, Pune University, Pune, Mahrashtra, India
}

\begin{abstract}
Problem statement: Oral cancer is one of the most common life threatening cancers all over the world, in particular Asian countries and tobacco is considered to be the most potent risk factor for oral cancer. This study was conducted to investigate the risk factors for oral cancer among the subjects from the studied area. Approach: A case-control study of 350 cases and 350 controls over a period of 19 months during April 2005 and September 2006 was carried out. The self reported information about their tobacco, alcohol along with other associated habits was collected by structured questionnaires. The consumption of tobacco was classified into three types, active smoking, passive smoking and smokeless form of tobacco. Results: There was a significant association between consumption of tobacco and the development of oral cancer ( $\mathrm{p}<0.05$ for all). Active smoking, in particular bidi smoking showed strong association with oral cancer compared to the passive smoking. Of the smokeless tobacco type, gutkha and tobacco flakes consumption showed the strong association. However, betel leaf and paan parag chewing had no association. While, alcohol consumption was associated with oral cancer with strongest determinant being the consumption of hard liquor. Dietary habits, in particular the non-vegetarian diet was significantly associated with oral cancer. The entire associations were statistically adjusted for possible confounders like age, gender, alcohol, the use of other tobacco types, non-vegetarian diet, education, location and monthly household income as appropriate. Conclusion: Smokeless tobacco consumption emerged as the strongest risk factor for oral cancer.
\end{abstract}

Key words: Oral cancer, tobacco, gutkha, mishiri

\section{INTRODUCTION}

Tobacco is one of the most preventable causes of oral cancer. Although there is evidence that smoking (cigarette, cigar and pipe) is associated with oral cancer, the smokeless tobacco (often called chewing tobacco or spit tobacco) seems to be strongly associated with oral cancer (Haumschild and Haumschild, 2009). These findings are based primarily on the epidemiological association of tobacco use with increased incidence of oral cancer. Alcohol and diet have also been reported to be associated with oral Cancer. Kamangar et al. (2009) have reported that $75 \%$ of all oral cancers could be attributed to heavy alcohol and tobacco consumption.

In India, tobacco is one of the most important public health issues and used in various forms. In addition to smoking, use of Smokeless Tobacco (SLT), in a variety of forms, is widespread among both men and women (Pednekar et al., 2009). The most common form of tobacco use in India are traditional forms like betel leaf (paan), a combination of betel-leaf, areca nut, slaked lime, tobacco and condiments; combinations of ingredients are altered according to individual preferences, smoking bidi (hand rolled cigarette), chewing tobacco flakes with or without lime, tobacco tooth powder (mishiri, a black powder obtained by roasting and powdering tobacco, which is then applied to the gums using a finger). Besides, the use of new products, blends such as panmasala and gutkha, is increasing not only among men but also among children, teenagers and women, (Schulz et al., 2009). Use of all forms of tobacco has also been shown to be

Correspondence Author: Abdoul Hossain Madani, Department of Hygiene, Schools of Health, Hormozgan University of Medical Sciences, Bandarabbas, Hormozgan, Iran Tel: +989171664947 Fax: +987615564560 
associated with increased all-cause mortality in the Indian population (Gupta et al., 2005).

However, in India where chewing and smoking tobacco is practiced, there is a striking incidence of oral cancer- these cases account for approximately 50\% of all cancer cases (Schulz et al., 2009). As the distribution of tobacco consumption is not uniform, it is often found to be significantly higher among lower socioeconomic groups (Shankar et al., 2010). There is a little systematic investigation on how the consumption of tobacco types distributed in oral cancer patients in Pune, India. Thus in this study we investigate how tobacco consumption (in its smoking and smokeless form) is distributed among the cases and controls. Based on this distribution, we estimate the extent of risk associated to oral caner for various exposure measures of tobacco use.

\section{MATERIALS AND METHODS}

Study setting: The data presented in this report was obtained from a hospital based case-control study, conducted at Morbai Naraindas Budharani Cancer Institute, Pune, India, during a span of 19 months, starting from February 2005 to September 2006.

Study population: A total of 700 subjects $(350$ each, cases and controls) were selected using simple random sampling procedure. The data related to demographic status, occupational history, tobacco and alcohol drinking habits, as well as dietary habits was collected from subjects after taking their written informed consent. In this study, the information on the environmental smoking tobacco (passive smoking) was also collected. The entire information was recorded through personal interview and structured validated questionnaires.

Definition of cases and controls: Cases were the newly diagnosed patients of oral cancer aged above 18 years. The diagnosis was confirmed by histopathological investigations and classified by the standard International Classification of Diseases (ICD10) criterion. The controls were selected from the relatives, friends and caretakers of cases, who accompanied the patients at the hospital. The controls were apparently healthy and did not reportedly have cancer. The controls were age and sex matched to the cases.

Statistical methods: The data is presented as the numbers with percentage (prevalence). The significance of difference between the proportions of qualitative characteristics is tested using Chi-square test of independence of attributes. The multivariate associations of risk factors with oral cancer were tested using multiple logistic regression analysis. The quantitative risk assessment was done by calculating the Odds Ratios (OR) with 95\% confidence intervals. All the associations were adjusted for potential confounders like age, gender, alcohol, the use of other tobacco types, non-vegetarian diet, education, location and monthly household income as appropriate. The entire data was analyzed using a Statistical Package for Social Sciences (SPSS) version 13.

\section{RESULTS}

Univariate analysis: The subjects were categorized into ever/never habituated to use of different products. The habits included the use of tobacco, consumption of alcohol and dietary intake. The tobacco use was categorized to smoking and smokeless form. The smokers were further classified into active and passive smokers. In active smoking, users of all types; filtered cigarette, unfiltered cigarette and bidi smoking formed the sub-categories. While, for smokeless tobacco the sub-categories included crude products (tobacco flakes, mishiri and supari), along with blends and mixed products (gutkha, paanmassala and betel leaf). Generally, blends are mixture of ingredients with or without tobacco, wherein, panmassala is a mixture (areca nut, catechu, cardamom, lime and number of fine natural perfuming materials) without tobacco while gutkha is a panmassala with tobacco. For alcohol habits subjects were classified into consumers of beer, wine, hard liquor and country made liquor. The dietary habit included vegetarian and non-vegetarian diets.

Table 1 shows the distribution of subjects by selected habits. The unadjusted ORs along with 95\% CIs are also shown for these individual consumption types.

In general, consumption of tobacco-in any form (chewing, active and passive smoking) was significantly different between cases and controls $(\mathrm{p}<0.001)$. Similarly, overall drinking alcohol habit and non-vegetarian diet were also significantly different between the two groups ( $\mathrm{p}<0.000$ for both) (Table 1).

In case of both active and passive smoking, the prevalence was significantly higher in cases compared to controls $(\mathrm{p}<0.001$ for both). In the active smoking sub categories viz., filtered cigarette, non-filtered cigarette and bidi (hand-rolled locally available cigarette), the prevalence was significantly larger in cases compared to controls ( $<<0.001$ for all). The bidi smokers had the higher risk of oral cancer. 
Am. J. Pharm. \& Toxicol., 5 (1): 9-13, 2010

Table 1: Distribution of subjects by selected habits (Univariate)

\begin{tabular}{|c|c|c|c|c|c|}
\hline Factors & Cases (350) n (\%) & Controls (350) n (\%) & $\mathrm{OR}^{*}$ & $95 \%$ CI of OR & p-value \\
\hline Tobacco Use (overall) & $328(93.7)$ & $200(57.1)$ & 11.2 & $7.0-18.0$ & 0.001 \\
\hline Active smoking (overall) & $125(35.7)$ & $61(17.4)$ & 2.6 & $1.8-3.7$ & 0.001 \\
\hline Filtered cigarette & $44(12.6)$ & $33(9.4)$ & 1.4 & $0.9-2.3$ & 0.149 \\
\hline Non filtered cigarette & $15(4.3)$ & $6(1.7)$ & 2.5 & $1.0-6.7$ & 0.046 \\
\hline Bidi (Hand rolled cigarette) & $70(20.0)$ & $20(5.7)$ & 4.1 & $2.4-6.9$ & 0.001 \\
\hline Passive smoking & $75(21.4)$ & $19(5.4)$ & 1.7 & $1.6-2.2$ & 0.001 \\
\hline Smokeless tobacco (overall) & $303(86.6)$ & $158(45.1)$ & 7.8 & $5.4-11.4$ & 0.001 \\
\hline Tobacco flakes & $175(50.0)$ & $55(15.7)$ & 5.3 & $3.7-7.6$ & 0.001 \\
\hline Betel leaf (Paan) & $44(12.6)$ & $31(8.9)$ & 1.4 & $0.9-2.4$ & 0.112 \\
\hline Pan parag & $21(6.0)$ & $18(5.1)$ & 1.1 & $0.6-2.2$ & 0.621 \\
\hline Gutkha & $112(32.0)$ & $21(6.0)$ & 7.3 & $4.5-12.0$ & 0.001 \\
\hline Supari & $32(9.1)$ & $12(3.4)$ & 2.8 & $1.4-5.6$ & 0.002 \\
\hline Mishri & $118(33.7)$ & $65(18.6)$ & 2.2 & $1.5-3.1$ & 0.001 \\
\hline Alcohol (overall) & $106(30.3)$ & 48 (13.7) & 2.7 & $1.8-3.9$ & 0.001 \\
\hline Beer & $28(8.0)$ & $14(4.0)$ & 2.1 & $1.1-4.1$ & 0.026 \\
\hline Wine & $4(1.1)$ & $6(1.7)$ & 0.6 & $0.2-2.3$ & 0.524 \\
\hline Hard liquor & $29(8.3)$ & $10(2.9)$ & 3.1 & $1.4-6.4$ & 0.002 \\
\hline Country liquor & $55(15.7)$ & $25(7.1)$ & 2.4 & $1.5-4.0$ & 0.001 \\
\hline Diet vegetarian & $52(26.4)$ & $145(73.6)$ & 0.2 & $0.1-0.3$ & 0.001 \\
\hline Non-vegetarian & $298(59.2)$ & $205(40.8)$ & 4.0 & $2.8-5.8$ & \\
\hline
\end{tabular}

*: Un-adjusted (crude) odds ratios

Table 2: Association of types of smoking and smokeless tobacco with oral cancer (multivariate-adjusted)

\begin{tabular}{|c|c|c|c|c|c|c|}
\hline \multirow[b]{2}{*}{ Factors } & \multicolumn{6}{|c|}{ Multivariate models (adjusted) } \\
\hline & $\begin{array}{l}\text { Model-1 OR } \\
(95 \% \text { CI })\end{array}$ & $\begin{array}{l}\text { Model-2 OR } \\
(95 \% \mathrm{CI})\end{array}$ & $\begin{array}{l}\text { Model-3 OR } \\
(95 \% \mathrm{CI})\end{array}$ & $\begin{array}{l}\text { Model-4 OR } \\
(95 \% \mathrm{CI})\end{array}$ & $\begin{array}{l}\text { Model-5 OR } \\
(95 \% \text { CI })\end{array}$ & $\begin{array}{l}\text { Model-6 OR } \\
(95 \% \text { CI })\end{array}$ \\
\hline \multicolumn{7}{|l|}{ Smoking tobacco } \\
\hline Filtered cigarette & $3.1(1.7-5.6)$ & $2.7(1.5-5.0)$ & $2.8(1.5-5.2)$ & $2.9(1.6-5.3)$ & $3.4(1.8-6.2)$ & $3.0(1.6-5.6)$ \\
\hline Non-filtered cigarette & $4.3(1.4-3.7)$ & $3.3(1.1-10.3)$ & $3.8(1.2-12.5)$ & $4.2(1.3-13.2)$ & $3.8(1.2-12.5)$ & $4.3(1.4-13.7)$ \\
\hline Bidi & $5.2(2.8-9.6)$ & $4.8(2.6-9.0)$ & $4.8(2.6-8.9)$ & $5.5(2.9-10.2)$ & $3.7(1.9-6.9)$ & $5.2(2.8-9.7)$ \\
\hline \multicolumn{7}{|l|}{ Smokeless tobacco } \\
\hline Tobacco falakes & $7.8(5.1-12.1)$ & $7.6(4.9-11.9)$ & $6.6(4.3-10.2)$ & $8.3(5.4-13.0)$ & $6.1(3.9-9.5)$ & $7.9(5.1-12.3)$ \\
\hline Gutkha & $12.0(6.6-21.7)$ & $12.7(7.0-23.2)$ & $12.8(7.0-23.7)$ & $12.1(6.7-21.8)$ & $12.4(6.8-22.5)$ & $12.0(6.6-21.7)$ \\
\hline Supari & $6.3(2.9-14.0)$ & $6.1(2.7-13.9)$ & $6.5(2.8-15.2)$ & $6.6(3.0-14.8)$ & $6.0(2.6-13.4)$ & $6.4(2.9-14.2)$ \\
\hline Mishiri & $3.0(1.9-4.8)$ & $3.0(1.9-4.9)$ & $2.7(1.7-4.3)$ & $3.3(2.1-5.4)$ & $2.6(1.6-4.1)$ & $3.0(1.9-4.9)$ \\
\hline Betel leaf & $1.9(1.0-3.4)$ & $1.8(1.0-3.3)$ & $1.7(0.9-3.2)$ & $1.9(1.1-3.6)$ & $1.6(0.9-2.9)$ & $1.9(1.0-3.5)$ \\
\hline Pan parag & NA & NA & NA & NA & NA & NA \\
\hline
\end{tabular}

Model-1: Adjusted for age, gender, other tobacco types; Model-2: Adjusted for model-1 plus alcohol; Model-3: Adjusted for model-1 plus nonvegetarian; Model-4: Adjusted for model-1plus location; Model-5: Adjusted for model-1 plus education; Model-6: Adjusted for model-1 plus occupation; NA: Not Available because of inadequate information

Similarly, the prevalence of almost all types of smokeless tobacco was significantly higher in cases ( $<<0.001$ for all) (Table 1). Of the types, chewing of gutkha was more common among the cases and had the higher risk of oral cancer. The next significant risk factor was the chewing of tobacco flakes. The use of paan-parag and betel leaf was not significant risk factor for oral cancer.

For the types of alcoholic beverages, country liquor, hard liquor and beer were significantly more common among cases ( $\mathrm{p}<0.05$ for all) (Table 1). So, also was the difference observed in the dietary habits of the two groups.

Univariate analysis revealed that the overall tobacco use was highly significant risk factor for oral cancer with OR of 11.2, (95\% CI of OR, 7.2-18.0), followed by non-vegetarian and alcohol consumption, 4.0 (2.8-5.8) and 2.7 (1.8-3.9) respectively. Whereas, the vegetarian dietary habit was found to be a protective factor for oral cancer 0.2 (0.1-0.3) (Table 1).

Multivariate analysis: Table 2 shows the multivariate Odds Ratios (OR) for smoking and smokeless tobacco use as an exposure through different multivariate models for adjustment of possible confounders like age, gender, tobacco types, alcohol, non-vegetarian habit, location of residency, education and occupation as appropriate (adjusted OR).

From Table 1 and 2 it is apparent that the multivariate and unadjusted risk assessment is different for various types of tobacco use. The unadjusted risk for filtered, cigarette smoking was not 
significant $(\mathrm{OR}=1.4 ; 0.9-2.3)$, but the multivariate risk ranged from 2.7-3.4 after adjusting for other appropriate risk factors through model 1-6. The unadjusted risk for non-filtered cigarette smoking was 2.5 (1.0-6.7), the multivariate risk ranged from 3.3-4.3 after adjusting for other appropriate risk factors through models 1-6.

The multivariate risk for bidi smoking ranged from 3.7-5.5 through models 1-6. While the unadjusted risk was 4.1 (Table 2).

Whereas, in smokeless types, while the crude risk of oral cancer for tobacco flakes was $5.3(\mathrm{p}<0.05)$, the multivariate risk for tobacco flakes increased significantly (range: 6.1-8.3). The un-adjusted risk for gutkha was 7.3, while, the multivariate risk ranged from12.0-12.8.

The multivariate risk of oral cancer with supari ranged from 6.0-6.5, whereas, the crude risk of oral cancer for supari was 2.8 .

The unadjusted risk for mishiri was 2.2 , whereas, the multivariate risk ranged between 2.6-3.3 after adjusting for other appropriate risk factors. The unadjusted for betel leaf was 1.4, which was not significant, whereas, the multivariate risk ranged between 1.6-1.9 after adjusting for other appropriate risk factors through models 1-6, it was not significant risk only in models 3 and 5. Paan parag was not significantly associated either in univariate or in multivariate models.

\section{DISCUSSION}

In this study we found that approximately $94 \%$ of patients with oral cancer have used tobacco products and the relative risk of developing oral cancer was 11 times that of the risk in never use tobacco. All forms of tobacco use (active and passive smoking as well as smokeless tobacco) were associated with oral cancer. However, a maximum risk was found among the smokeless tobacco users $\mathrm{OR}=7.8$ (5.4-11.4). (Muwonge et al., 2008) and (Znaor et al., 2003) have reported that the smokeless tobacco to be the higher risk factor than smoking tobacco (OR 5.05), however it is about two fold less risk compared to our study. It is interesting to note that of the smokeless tobacco consumption, the risk was strongly determined by gutkha followed by tobacco flakes consumption. It is probably due to the combined effect of the ingredients (tobacco, areca nut, catechu, cardamom, lime and number of fine natural perfuming materials) present in gutkha. In the last few decades, small, attractive and inexpensive sachets of betel leaf substitutes (gutkha and panmasala) have become widely available.
Aggressively advertised and marketed, often claimed to be safer products, they are consumed by the very young and old alike, particularly in India (Nair et al., 2004). Next risk determining factor was found to be tobacco flakes (OR 5.6), which is used with or without lime and kept in the mouth for different duration of time depending on the personal habits. Of the other smokeless tobacco types, Mishiri showed relatively lower risk than tobacco flakes. This could be because of the difference in processing and use. Mishiri is applied on teeth and gums and spit out within a relatively short time. While tobacco flakes are kept for a longer time, resulting in differential release and absorption of toxic components of tobacco. Earlier case control studies from Mumbai have identified betel leaf chewing as major risk factor for oral cancer in India (Nair et al., 2004). The absence of effect for betel leaf in the present study may be due to the predominant habits of spitting out the quid and its extracts with saline rather than swallowing, which thus prevented carcinogens contact with oral cavity epithelium. Similar results have also been reported in one of the earlier study conducted in (Thomas et al., 2007)

Smoking was a risk but it appears to be the relatively weak risk factor for oral cancer in particular. According to our results, daily smokers have about 3 fold increased risk compared to never-smokers, which is supported by findings of Thomas et al. (2007) in Papua New Guinea. We found an increased risk of oral cancer for bidi smokers compared to never smokers (OR (95\%CI) 4.1 (2.4-6.9), whereas no significant pattern of risk was found for cigarette smokers. Such results have been reported by Rahman et al. (2003); Warnakulasuriya (2005) and Subapriya et al. (2006). It may be due to the higher content of nicotine in bidi. According to Malson et al. (2001); (2002), the nicotine concentration in bidi is $21.2 \mathrm{mg} \mathrm{g}^{-1}$ compared to commercial filtered cigarette $\left(16.3 \mathrm{mg} \mathrm{g}^{-1}\right)$ and unfiltered cigarette $\left(13.5 \mathrm{mg} \mathrm{g}^{-1}\right)$. In addition, bidi is prepared by rolling tobacco in dried leaf of tendu (Diospyros malanoxylon) or Temburi tree (Diospyros ebenum) in comparison to US cigarettes, the mainstream smoke of bidi contains a much higher concentration of several toxic agents such as hydrogen cyanide, carbon monoxide, ammonia, other volatile phenols and carcinogenic hydrocarbons such as benz(a)anthracen and benozopyrene (Pednekar et al., 2009). Moreover in India, bidi smoking being affordable to mass of population is most common than cigarette smoking. This aspect may explain bidi being a factor for increased risk of oral cancer in India.

Smokeless tobacco has a stronger effect than a smoking type because of the direct contact of the tobacco carcinogens with the oral epithelium as the 
chewing tobacco products are chewed or kept in the mouth. However, the etiologic role of these factors is not well understood and further methods for modifying them need to be developed (Weinberg and Stefan, 2002). The insignificance either in univariate or in multivariate models for pan parag may have been the result of inadequate data in this study.

On multivariate analysis, the smoking and smokeless forms of tobacco predicted oral cancer significantly and independently after adjusting for the other types of tobacco use, alcohol, age, gender, location, education and occupation. The smokeless form of tobacco emerged as a strong independent risk factor for oral cancer. It is interesting to note that there is a difference in the multivariate risk and uni-variate risk assessment of each factor. This suggests that there is a significant effect of the confounders like age, gender, education, occupation on the use all types of tobacco.

\section{CONCLUSION}

In conclusion, smokeless tobacco consumption emerged as the strongest risk factor for oral cancer. Further studies are required in other parts of India to demonstrate the similar effects of tobacco consumption also to find out the actual prevalence of oral cancer to get an idea of the burden of the underlying health problem. There is a need for appropriate prevention and planning strategies for tobacco consumption. The local government initiatives towards enforcing of the programs to improve the education and economic status as well as create awareness about the oral cancer and its damaging consequences would be most desirable.

\section{REFERENCES}

Gupta, P.C., M.S. Pednekar, D.M. Parkin and R. Sankaranarayanan, 2005. Tobacco associated mortality in Mumbai (Bombay) India. Results of the Bombay cohort study. Int. J. Epidemiol., 34: 1395-402. PMID: 16249218

Haumschild, M.S. and R.J. Haumschild, 2009. The importance of oral health in long-term care. J Am. Med. Dir. Assoc., 10: 667-71. PMID: 19883892

Kamangar, F., W.H. Chow, C.C. Abnet and S.M. Dawsey, 2009. Environmental causes of esophageal cancer. Gastroenterol. Clin. North Am., 38: 27-57. PMID: 19327566

Malson, J.L., K. Sims, R. Murty and W.B. Pickworth, 2001. Comparison of the nicotine content of tobacco used in bidis and conventional cigarettes. Tobacco Control, 10: 181-183. PMID: 11387541

Malson, J.L., E.M. Lee, E.T. Moolchan and W.B. Pickworth, 2002. Nicotine delivery from smoking bidis and an additive-free cigarette. Nicotine Tobacco Res., 4: 485-490. PMID: 12521408
Muwonge, R., K. Ramadas, R. Sankila, S. Thara and G. Thomas et al., 2008. Role of tobacco smoking, chewing and alcohol drinking in the risk of oral cancer in Trivandrum, India: A nested case-control design using incident cancer cases. Oral Oncol., 44: 446-454. PMID: 17933578

Nair, U., H. Bartsch and J. Nair, 2004. Alert for an epidemic of oral cancer due to use of the betel quid substitutes gutkha and pan masala: A review of agents and causative mechanisms. Mutagenesis, 19: 251-262. PMID: 15215323

Pednekar, M.S., J.R. Hébert and P.C. Gupta, 2009. Tobacco use, body mass and cancer mortality in Mumbai cohort study. Cancer Epidemiol., 33: 424-30. PMID: 19854693

Rahman, M., J. Sakamoto and T. Fukui, 2003. Bidi smoking and oral cancer: A meta-analysis. Int. J. Cancer., 106: 600-604. PMID: 12845659

Schulz, M., P.A. Reichart, C.A. Ramseier and M.M. Bornstein, 2009. Smokeless tobacco: A new risk factor for oral health? A review. Schweiz Monatsschr Zahnmed, 119: 1095-1109. PMID: 20020590

Shankar, A., A. McMunn, A. Steptoe, 2010. Healthrelated behaviors in older adults relationships with socioeconomic status. Am. J. Prev. Med., 38: 39-46. PMID: 20117555

Subapriya, R., A. Thangavelu, B. Mathavan, C.R. Ramachandran and S. Nagini, 2006. Assessment of risk factors for oral squamous cell carcinoma in Chidambaram, Southern India: A case-control study. Eur. J. Cancer Prev., 16: 251-256. PMID: 17415096

Thomas, S.J., C.J. Bain, D. Battistutta, A.R. Ness and D. Paissat et al., 2007. Betel quid not containing tobacco and oral cancer: A report on a case-control study in Papua New Guinea and a meta-analysis of current evidence. Int. J. Cancer, 120: 1318-1323. PMID: 17163423

Warnakulasuriya, S., 2005. Bidi smokers at increased risk of oral cancer. Evid. Based Dent., 6: 19. PMID: 15789047

Weinberg, M.A., D.J. Estefan, 2002. Assessing oral malignancies. Am. Fam. Phys., 65: 1379-1384. PMID: 11996421

Znaor, A., P. Brennan, V. Gajalakshmi, A. Mathew and V. Shanta et al., 2003. Independent and combined effects of tobacco smoking, chewing and alcohol drinking on the risk of oral, pharyngeal and esophageal cancers in Indian men. Int. J. Cancer, 105: 681-686. PMID: 12740918 